\title{
THE DANGEROUS SUBMUCOUS UTERINE MYOMA
}

\author{
A. E. R. BuCKLE, F.R.C.S., M.R.C.O.G. \\ Department of Obstetrics and Gynacology, Lewisham General Hospital, London, S.E.13.
}

Although the least commonly encountered group, submucous uterine myomata attract early attention clinically because of encroachment on the uterine cavity. Symptoms include menorrhagia, intermenstrual bleeding and discharge and, because they may become polypoidal and be extruded from the uterus, pain may be a prominent feature. The ease of entry of microorganisms from the genital tract makes this form of myoma prone to infection, particularly after pregnancy or abortion.

Earlier-sought advice, diagnosis and treatment mean that the more severe cases will nowadays be less commonly encountered. Nevertheless, the complications of extrusion, infection, necrosis and bleeding may occur at any age, both after delivery or apart from pregnancy, as the following cases illustrate :

\section{Case No. 1. Submucous myoma complicating pregnancy, labour and the puerperium.}

Mrs. G.P., aged 28 years, gravida 4, para 2, was referred to the ante-natal clinic with a persisting oblique breech lie of the foetus at 35 weeks gestation. There was no relevent medical or obstetric history.

In the present pregnancy there had been a severe degree of anæmia (hæmoglobin $7.1 \mathrm{~g} . / 100 \mathrm{ml}$.) and investigation had revealed a minor degree of iron deficiency, the bone marrow biopsy being normal. There was no evidence of chronic renal tract infection. Treatment with oral iron and, later, with folic acid had resulted in some improvement but this was not complete and at the time of her referral to the clinic, the hæmoglobin level was $10.8 \mathrm{~g} . / 100 \mathrm{ml}$.

Abdominal examination confirmed the period of gestation and, although the foetus could be turned to the oblique vertex position, it quickly reverted to its original position. Because of the possibility that the malpresentation resulted from a low-lying placenta, the patient was advised admission; she was admitted in premature labour before this could be arranged. The foetus was at this time presenting by the breech; labour proceeded rapidly, an assisted breech delivery being performed. The total blood loss at delivery was 200 millilitres.

The infant, a live male, weighted $2.3 \mathrm{~kg}$. ( 5 pounds) and was limp and cyanosed at birth. Although showing initial improvement, a pan-systolic precordial murmur, maximal in the left second interspace, was present and the femoral pulses were not palpable. Congestive cardiac failure developed and the infant died 42 hours after delivery, the clinical diagnosis being coarctation of the aorta. Autopsy revealed atresia of the aortic valve and hypoplasia of the ascending aorta.

The patient was discharged home to the care of her general practitioner 48 hours after delivery only to be re-admitted 15 days later with a history of continuing vaginal loss and associated abdomina pain. On examination, the patient was febrile (temperature $39^{\circ} \mathrm{C} ; 102^{\circ} \mathrm{F}$ ), pale and in pains Abdominal examination showed the uterus to be tender and palpable halfway between pubis and the umbilicus and vaginal examination revealed the cervix to be three fingers dilated, a mass being felth through the cervix on the left postero-lateral surfaces of the uterus. A diagnosis of a degenerating subeo mucous uterine myoma was made.

Investigations: Blood group ' $\mathrm{O}$ ': $\mathrm{Rh}+, \mathrm{Hb}$ 7.9 g./ $100 \mathrm{ml}$., WBC 14,000 cu. mm. (polys: 10,500). Chest X-ray normal. Blood culture negative. High vaginal swab-coliform organisms, sensitive to tetracycline and chloramphenicol.

Treatment: The patient was given tetracycline, $25 \overrightarrow{0^{\circ}}$ mg. 6-hourly for five days and was transfused with two pints of blood. The temperature settled but the abdominal pain persisted and became more severe four days after admission. Vaginal examination ato this time showed the cervix to be widely dilated withe the myoma protruding into the upper vagina? Operative removal was undertaken. Vaginal enuclgaee tion was attempted but the myoma was found ago have a sessile attachment to the uterus and attempt was abandoned; abdominal hysterectomy ovarian conservation was performed, operation bemgs uneventful. The post-operative course was uneventfue and the patient discharged from hospital on the्ष sixteenth post-operative day. Subsequent follow-up at the out-patient clinic revealed no abnormality an\& she was discharged from the care of the hospital.

Histological report: Projecting into the cavity of the uterus is a large ragged mass of partly necrotic and hæmorrhagic tissue some $12 \times 10 \times 7 \mathrm{~cm}$. which has the consistency of a fibromyoma. Microscopica? examination shows extensive areas of necrosise hæmorrhage and œdema with polymorpho-nucleas infiltration. There is no evidence of malignancy. The endometrium shows an endometritis.

\section{Comment}

Although pregnancy is not infrequently encountered in patients with intramural or sub peritoneal myomata, it is rare with the submucous variety. Parks and Barter (1952) emphasised that the latter will produce major problems both froms the point of diagnosis and of treatment. The occurrence of tumour degeneration and infection after abortion may necessitate hysterectomyeț prolonged bleeding and pyrexia in the puerperium in a patient with uterine myomata indicate infection of a submucous form and may require abdominal hysterectomy. Cody and Wall (1956\% reported a case of an infected submucous myoma which extruded from the uterus three weeks afte $\bar{b}$ delivery, vaginal enucleation being successfull 


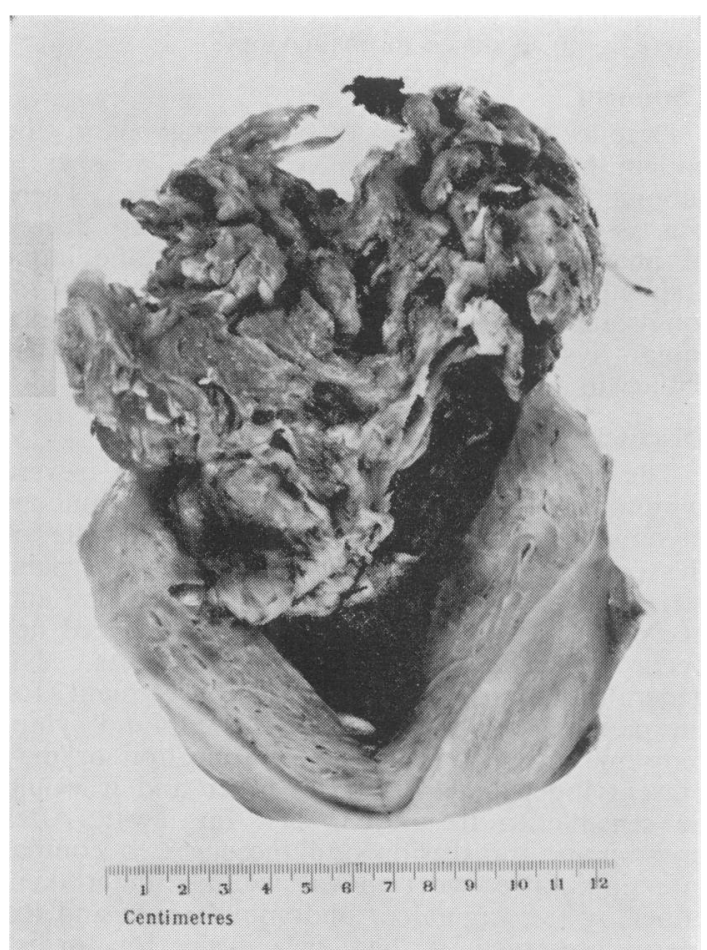

FIG. 1.-CASE 1: Hysterectomy specimen showing the large necrotic submucous myoma.

accomplished. Abitbol (1957) reviewed 14 cases of submucous myomata in pregnancy encountered over a 30-year period. Diagnosis was made during post-partum curettage for abnormal bleeding ( 5 cases; 2 required hysterectomy), after partial expulsion of the myoma (4 cases, all treated by avulsion), because of post partum morbidity ( 2 cases, both treated by hysterectomy) or during Cæsarean section (3 cases, all treated by hysterectomy). Abitbol also pointed out that in the case of the large myoma infection may persist for many weeks and if the temperature does not settle rapidly, it is sensible to proceed to hysterectomy.

In the described case, the presence of the myoma was responsible for the malpresentation of the foetus and it is likely that degenerative change in the tumour was responsible for the anæmia discovered during pregnancy.

Case No. 2. Submucous myoma: no referable previous symptoms.

Mrs. V.O'C., aged 29 years, para 1, admitted with a history of heavy vaginal bleeding for nine days. The patient gave a history of a duodenal ulcer dating from the age of 13 years. She had had medical treatment for this and had been almost symptom free for the previous 7 years. Her pregnancy two years prior to the present admission had been uneventful, but she had been noted to have two small subperitoneal fibroids at the post-natal examination.
The present episode of vaginal bleeding had occurred 26 days after a normal period, the loss being heavy with the passage of clots but there had been no pain.

On examination the patient was febrile (temperature $39.2^{\circ} \mathrm{C} ; 102.4^{\circ} \mathrm{F}$ ) and extremely pale. The blood pressure was normal and there was a soft systolic murmur at the apex. The chest was clear and there was no abnormality on abdominal examination. Vaginal examination revealed the cervix to be two fingers dilated and a sessile mass was palpable on the left side of the cervical canal, some $4 \mathrm{~cm}$. in width; the upper edge could not be reached. A steady bright red blood loss was occurring from the cervical canal. A diagnosis of an infected extruding submucous myoma of the uterus or upper part of the cervical canal was made.

Investigations: Blood group 'A'; Rh. + , Hb. 4.6 g. $/ 100 \mathrm{ml}$. WBC $11,000 /$ per cu. $\mathrm{mm}$. Chest X-ray normal. High vaginal swab grew diphtheroids and scanty streptococcus frecalis.

Treatment: A slow intravenous infusion of blood was commenced and, after 4 pints had been given, the hæmoglobin had risen to $9.6 \mathrm{~g} . / 100 \mathrm{ml}$. The vaginal loss ceased after 24 hours. Two further pints of blood were given prior to operation. Examination in the operating theatre confirmed the presence of a large sessile submucous myoma on the left posterior wall of the cervix and lower part of the body of the uterus, although it was not possible to feel the upper edge. After incision through the capsule, the myoma was enucleated in several fragments. Limited access made it impossible to identify bleeding points in the capsule of the myoma so recourse was had to packing the bed of the tumour with ribbon gauze. The post-operative course was uneventful and no undue loss followed the removal of the pack some 48 hours after operation. The patient was discharged from hospital 10 days after operation. Follow-up in the out-patient clinic showed a return to normal in the size of the cervix and there was a return to normal menstrual cycle. Stool examination was carried out for gastro-intestinal bleeding, with a negative result; a barium meal showed a characteristic ulcer deformity of the duodenal cap. At the time of discharge from the out-patient clinic, the hæmoglobin was $11.5 \mathrm{~g} . / 100 \mathrm{ml}$.

Histological report: The tumour pieces are part of a benign myoma, the total weight being $114 \mathrm{~g}$. There is no evidence of malignancy.

\section{Comment}

Heavy vaginal bleeding of the degree encountered in this instance may well have resulted from surface necrosis of the tumour with the subsequent rupture of vessels. Tumours of this type are rarely encountered, especially at this age, and vaginal enucleation was attempted as it appeared to be accessible from the vaginal route. Had it not been possible to arrest hæmorrhage from the tumour bed, an abdominal approach would have been necessary to display the affected area. It is of interest that the patient had at no time had abdominal pain, despite the dilatation of the cervix.

Case No. 3. Submucous myoma: previous symptoms referable to myomata.

Mrs. G.L., aged 47 years, para 3, was admitted complaining of a heavy and offensive blood loss per 
vaginam for two weeks. The relevent history was of admission for the investigation of an iron-deficiency anæmia 4 years previously. Examination at that time had shown no other cause for the anæmia than menorrhagia from uterine myomata and she was advised but refused operation. She denied that there had been any undue loss with her periods from that time until the present episode, and also denied having any abdominal pain.

On examination, the patient was febrile (temperature $39.5^{\circ} \mathrm{C} ; 103^{\circ} \mathrm{F}$ ), extremely pale and appeared toxic. There was smoothness of the tongue, kolonychia and there was a systolic murmur at the apex of the heart. The chest appeared normal. Examination of the abdomen showed there to be a mass arising out of the pelvis equivalent in height to a sixteen-week pregnancy. Vaginal examination revealed the upper vagina filled by a large, discoloured, necrotic mass, the cervix not being identified. A diagnosis of an infected extruded submucous myoma in association with long-standing anæmia was made.

Investigations: Blood group 'A'; Rh. + , Hb. 3.8 g./ $100 \mathrm{ml}$., WBC 15,000/cu. mm. (Neutrophils $91 \%$ ). Chest X-ray normal. Blood culture negative.

Treatment: Slow blood transfusion was commenced, five pints being given over four days. There was a marked improvement in the clinical condition, the temperature fell to normal and the hæmoglobin rose to $10 \mathrm{~g} . / 100 \mathrm{ml}$.

Operation was carried out 6 days after admission, examination under anæsthesia confirming the presence of a large, necrotic myoma in the upper vagina, the cervix being felt widely dilated around the upper part of the mass. In view of the age of the patient, total abdominal hysterectomy was carried out, the ovaries also being removed. Operation was uneventful and the patient was given two further pints of blood during the procedure. Tetracycline, $250 \mathrm{mg}$. 6-hourly, was given for the five days following operation. Her course was uneventful and she was discharged three weeks later. Follow-up at the out-patient clinic revealed her to be well and symptom-free. The hæmoglobin was $12.8 \mathrm{~g} . / 100 \mathrm{ml}$. and she was discharged from the care of the hospital.

Histological report: The uterus contains a large fleshy myoma, $15 \times 10 \times 9 \mathrm{~cm}$., which is arising by a broad base from the posterior wall of the uterus. The mass, the surface of which is blackened and necrotic, is lying partly within the cavity of the uterus and partly through the widely dilated cervix. Microscopically, there is marked œedema and leucocyte infiltration and some vessels contain recent thrombi. There is no evidence of malignancy.

\section{Comment}

Despite the absence of abdominal pain, thec patient had extruded this very large, degenerate $\Rightarrow$ submucous myoma through the cervix. Theres was associated systemic upset and a gross degreeo of anæmia, although it was felt that the lattero resulted from recent blood loss superimposed on $\overline{\bar{n}}$ long-standing anæmia from menorrhagia. Abdo- $\bar{\varnothing}$ minal hysterectomy was here the treatment of $\mathcal{O}$ choice in view of the age of the patient.

\section{Discussion}

The above three cases emphasise the severe $\overrightarrow{\vec{H}}$ symptoms which may result from submucous $\omega_{\sigma}$ myomata of the uterus and justify the title of this paper. Although classically there will be pain, hæmorrhage and pyrexia from infection and necrosis of the tumour, such symptoms need not $\stackrel{\oplus}{-}$ occur together, as the cases illustrate. Nof generalisation can be made about treatment, for the patients may be of any age group and symp- $\vec{A}$ toms may follow pregnancy or abortion or mayos present independently of the latter and it would? be sensible to treat each case on merit. Afterpreliminary transfusion and measures to combat 3 infection, operative removal will be undertaken, either by the vaginal or abdominal route and the $\mathcal{A}$ uterus preserved, if there is a desire for further $\vec{\theta}$ pregnancies, in the younger age group; otherweos it would seem reasonable to proceed to hysteres. tomy.

\section{Summary}

Three cases in which severe symptoms were⿳亠口冋े associated with a submucous uterine myoma are described.

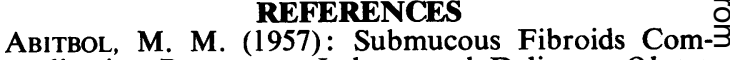
plicating Pregnancy, Labour and Delivery, Obstet. Gynec., 10, 529.

CoDY, M. L., and Wall, H. A. (1956): Submucous Uterine Myoma Causing Postpartum Complications, $\frac{\widehat{3}}{3}$ Obstet. Gynec., 7, 304.

PARKE, J., and BARTER, R. H. (1952): The Myomatous Uterus Complicated by Pregnancy, Amer. J. Obstet Gynec., 63, 260.

\section{THE SPLENIC FLEXURE SYNDROME

\author{
J. ShaFAR, M.D., M.R.C.P., D.P.H. \\ Consultant Physician, \\ Burnley and District Group of Hospitals
}

THE SPLENIC-FLEXURE syndrome would appear to have received inadequate attention as judged by the paucity of reports in the literature and by its infrequent inclusion in textbooks of general medicine and even of disorders of the alimentary tract. Familiarity with the condition is important not only in elucidation of the nature of some examples of upper abdominal pain, but also by reason of its simulation of pain of cardiac ore pulmonary origin. Palmer, Deutsch and Scotto (1955) were impressed by the common diagnostic? error of coronary artery disease; in many instanceso the subjects had become cardiac invalids as a con-o sequence of ill-directed medical advice. These 